\title{
CHOICE OF INTERLEAVERS FOR SPACE-DIVERSITY CODES IN HIPERMAN AND 802.16a BROADBAND WIRELESS SYSTEMS
}

\author{
Roshano Roberts, Reza Hoshyar, M. Sadegh Fazel, Rahim Tafazolli \\ Centre for Communication Systems Research (CCSR), \\ The University of Surrey \\ Guildford, Surrey, GU2 7XH, UK \\ email: \{r.s.roberts, r.hoshyar, m.fazel, r.tafazolli\}@eim.surrey.ac.uk
}

\begin{abstract}
In this paper, we study the choice of block interleavers in a MIMO-OFDM based broadband fixed wireless access system for the HIPERMAN and IEEE 802.16a standards with space-frequency trellis coding under typical channel conditions. It has prevously been shown that a given code offers varying levels of error performance depending on the degree of frequency selectivity of the channel. Code performance was comparatively better in channels with higher frequency selectivity or delay spreads, while performance was particularly degraded in channels with low frequency selectivity. In order to extract the maximum possible frequency diversity and close this gap in performance, we investigate the use of apropriate interleavers. As different channels favour interleavers of different depths, we recommend suitable interleaver depths for the typical channels considered. With these interleavers the performances can be significantly improved and can be made comparable despite the variation in channel frequency selectivity.
\end{abstract}

Keywords - Interleavers, space-frequency coding, MIMOOFDM, 802.16a, HIPERMAN.

\section{INTRODUCTION}

The use of the Internet as an everyday tool and the growing trend in the use of multi-media rich instant messaging have increased the demand for Broadband Fixed Wireless Access (BFWA) systems. To address the high data rate requirements in areas where deployment of Wireless Local Area Networks (W-LAN) is not practicable, two main standards for Metropolitan Area Networks (MAN) are being developed. The ETSI HIPERMAN (High Performance Radio MAN) and the IEEE 802.16a standards are fully harmonised standards that target the sub- $11 \mathrm{GHz}$ frequency range. Within these standards, the possibility to use the highly promising spatial diversity methods, such as space-time coding, are considered. One such method is the space diversity trellis coding, which offers diversity and coding gains when multiple transmit antennas are available. This is an attractive feature specially for downlink as the Base station can easily accommodate an antenna array of reasonable physical dimensions.

This work has been partly funded by the European Union through the 5 th framework project IST-STRIKE: Spectrally efficient fixed wireless networks based on dual standards (No. IST-2001-38354). http://ist-strike.org
Space diversity trellis codes were first introduced by Tarokh as space-time codes in the context of narrow-band systems [1]. Consequently, optimal codes for different channel and antenna conditions have been published in [2] and [3]. The application of space-diversity codes in the frequency domain for an Orthogonal Frequency Division Multiplexing (OFDM) system was addressed in [4], where they are employed along Tx antennas and OFDM sub-carriers as spacefrequency codes. It was further shown that the optimisation of these space-diversity codes, for space-time application does not gurantee good space-frequency codes. An important difference between the two applications from the fading perspective is that, in space-time application the fading along the code length is governed by the temporal fading pattern resulting from Doppler spread caused by the relative movement of the $\mathrm{Tx}$ or $\mathrm{Rx}$ with respect to the propagation medium. In most cases, when the code frame sizes are moderate and Doppler spread is not high, the fading will be approximately constant over a code frame, leading to the well known quasi-static fading assumption. However, in the space-frequency application, the fading along the code frame is heavily influenced by the frequency transfer function of the channel, which cannot be approximated as constant, specially in a broadband system.

In [5], the performance of the known optimum space-time codes of [2] and [3] employed as space-frequency codes is studied, and it is found that code performance was channel dependent such that the performance was worse in channels with small delay spreads (and thus less frequency selective), than in channels of high delay spreads (highly frequency selective), even if the number of taps and the powers were the same.

In this paper, we investigate the use of approptiate interleaving to extract the diversity resources available in the space-frequency channel, so that the gap in performance mentioned above can be closed. The interleavers operate on the spatial vector symbols such that the output label of the trellis is not altered and only the order in which they are transmitted over the channel is changed. The performances are compared using bit and packet (code-frame or codeblock) error rates, of these codes in an OFDM system based on the said standards. 
In the next section, we briefly introduce the space-diversity trellis codes and the interleaving method used. Subsequently, the transceiver system and channel models used are outlined. The results are then presented and discussed, followed by the conclusions reached.

\section{SPACE-DIVERSITY CODES}

\section{A. Space-Time codes}

Tarokh et al., first laid the foundations of space-time trellis coding and derived the design criteria for space-time codes in [1], where they showed that maximising the minimum rank and the minimum determinant of the distance matrix (defined below) over all code words would minimise the pair-wise error probability and consequently provide robust codes, for constant fading along the length of the code. When $\mathbf{C}=\left\{\mathbf{c}_{1}, \mathbf{c}_{2}, \cdots, \mathbf{c}_{l}\right\}$ and $\hat{\mathbf{C}}=\left\{\hat{\mathbf{c}}_{1}, \hat{\mathbf{c}}_{2}, \cdots, \hat{\mathbf{c}}_{l}\right\}$ are two codeword matrices, where $l$ is the length of a codeword; $\mathbf{c}_{t}=\left\{c_{t}^{1}, c_{t}^{2}, \cdots, c_{t}^{N_{t}}\right\}^{T}$ is the code vector transmitted at time instant $t ; c_{t}^{i}$ is the signal on transmit antenna $i$; and $N_{t}$ is the number of transmit antennas, the product distane matrix $\mathbf{A}$ is defined as:

$$
\mathbf{A}(\mathbf{C}, \hat{\mathbf{C}})=\mathbf{B}(\mathbf{C}, \hat{\mathbf{C}}) \mathbf{B}^{H}(\mathbf{C}, \hat{\mathbf{C}})
$$

where the superscript ()$^{H}$ denotes conjugate-transpose and $\mathbf{B}(\mathbf{C}, \hat{\mathbf{C}})$ is the difference matrix, which is expanded as:

$$
\mathbf{B}(\mathbf{C}, \hat{\mathbf{C}})=\left(\begin{array}{cccc}
c_{1}^{1}-\hat{c}_{1}^{1} & c_{2}^{1}-\hat{c}_{2}^{1} & \ldots & c_{l}^{1}-\hat{c}_{l}^{1} \\
c_{1}^{2}-\hat{c}_{1}^{2} & c_{2}^{2}-\hat{c}_{2}^{2} & \ldots & c_{l}^{2}-\hat{c}_{l}^{2} \\
\vdots & & \ddots & \vdots \\
c_{1}^{N_{t}}-\hat{c}_{1}^{N_{t}} & c_{2}^{N_{t}}-\hat{c}_{2}^{N_{t}} & \ldots & c_{l}^{N_{t}}-\hat{c}_{l}^{N_{t}}
\end{array}\right)
$$

Reference [2] has reported optimal codes that maximise the minimum rank and the minimum trace of the distance matrix $\mathbf{A}$ over all codewords since these are proved to be the dominant factors affecting the pair-wise error probability when the product of the number of $\mathrm{Tx}$ and $\mathrm{Rx}$ antennas is high ( $>4$ in practice) for constant fading along the length of the code (which corresponds to flat fading across the frequency bandwidth when used as space-frequency code).

In [3], optimal codes designed with Tarokh's criterion of maximising the minimum symbol hamming distance and minimum product distance over all code words, for Fastfading channels are reported. Fast-fading here is defined as independent fading along the length of the code (corresponding to independent fading of the OFDM sub-carriers in space-frequency configuration).

These codes are reported to have been found by exhaustive computer search over all possible code generator matrices of given size. We consider both these codes (optimal for Quasi Static channel and specially suited for high number

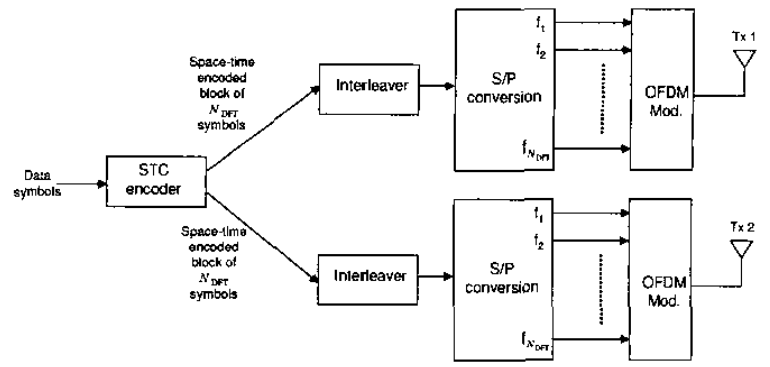

Fig. 1. MIMO-OFDM system with space diversity trellis coding.

of antennas, and the one suited for Fast Fading channel and for low number of antennas) as space-frequency codes for the HIPERMAN system. In [5], the performance of the above codes are evaluated in space-frequency configuration and it is noted that the performance depended quite heavily on the channel fading type when there was only one Rx antenna. It is also noted that the codes of [2] demonstrated an overall better performance. Thus, in this paper, we first study the performance enhancement with the 16-state codes of [2] (Vucetic 16-state) with one $\mathrm{Rx}$ antenna, and we consequently note that the same interleavers also enhance the performance with the codes of [3] (Firmanto 16-state). When there are more $\mathrm{Rx}$ antennas, the relative merit of each interleaver will still be the same. However, when more $\mathrm{Rx}$ antennas are available, the receive diversity dominates (than the diversity due to fading fluctuations) [5]. Nevertheless, the single $\mathrm{Rx}$ antenna case is an important one as most subscriber stations of BFWA services are envisaged to have only a single antenna [6].

\section{B. Vector Interleaving}

Interleaving is performed on the vector symbols $c_{k}$ (the sylmbols on sub-carrier $k$ over all Tx antennas) after space diversity encoding. When space-time vector symbols in a coded block are interleaved while preserving the integrity of the vector symbols, it is equivalent to the code matrix $\mathbf{C}$ being post multiplied by a permutation matrix $\mathbf{P}$. A permutation matrix of size $l \times l$ is obtained from permuting columns of the identity matrix of the same size and is thus a matrix repesentation of a permutation.

\section{SYSTEM DESCRIPTION}

\section{A. Simulation set-up and channel models}

The MIMO-OFDM transmitter is shown in Fig. 1 whose parameters are selected from the HIPERMAN standard [7], as summarised in Table $I$. It is a 256 subcarrier TDMAOFDM system with 192 data subcarriers.

The frequency selectivity of the channel was simulated using selected profiles from the Stanford University Interim (SUI) models as specified for BFWA systems in [8]. Two of these delay profiles (SUI-3 and SUI-5), that have very low Doppler 
Table I

Simulation system parameters.

\begin{tabular}{|c|c|}
\hline Parameter & Value \\
\hline Num. OFDM sub-carriers & $256(192$ data, 8 pilot, 56 null) \\
\hline $\begin{array}{c}\text { Num. OFDM symbols spanned } \\
\text { by the space-diversity code }\end{array}$ & 1 \\
\hline OFDM symbol duration & $160 \mu$ s in both channels \\
\hline Channel profiles and & SUI-3 (with GT fraction $=1 / 4)$ \\
Guard Time (GT) & SUI-5 (with GT fraction $=1 / 4)$ \\
\hline Bandwidth of OFDM signal & $1.75 \mathrm{MHz}$ \\
\hline Sampling Frequency & $2 \mathrm{MHz}$ \\
\hline
\end{tabular}

spreads representing low to no mobility applications such as BFWA [6], and a frequency-flat single tap Rayleigh fading channel were used for comparison. The SUI-3 channel is representative of a low delay spread case with $\tau_{r m s}=$ $0.264 \mathrm{~ms}$ (low frequency selectivity) and the SUI-5 channel model is a high delay spread channel with $\tau_{r m s}=2.842$ ms (high frequency selectivity). Typical examples of the frequency response of these channels are depicted in Fig. 2, where the distinctly contrasting fading behaviour across the frequency domain can be noted. The channel parameters are summarised in Table II. Fading processes are modelled with independent realisations between the antennas, representing good antenna separation and rich scattering propagation medium.

\section{B. Possible Block Interleavers}

Since the number of data carriers is 192 , the possible block interleaver sizes can be as in Table III. Out of these, the following sizes have been studied: $2 \times 96,4 \times 48,6 \times 32,8 \times 24$, $12 \times 16,16 \times 12,32 \times 6,48 \times 4,64 \times 3$ (all of the possibilities except $3 \times 64,24 \times 8$ and $96 \times 2$, as these are very close in structure to another one studied). A random interleaver

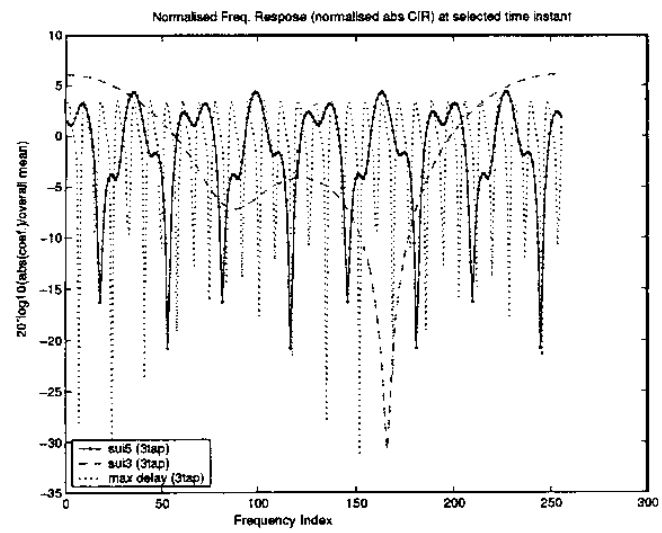

Fig. 2. Example snapshot of impulse responses of SUI-3 and SUI-5 channels and an artificial maximum delay channel of 3 uniformly spaced equal-power taps.
Table II

Channel Parameters.

\begin{tabular}{|c|c|c|c|c|}
\hline channel model & Parameter & Tap-1 & Tap-2 & Tap-3 \\
\hline SUI-3 & Delay ( $\mu \mathrm{s})$ & 0 & 0.4 & 0.9 \\
$\left(\tau_{r m s}=0.264 \mu \mathrm{s}\right)$ & Power (dB) & 0 & -5 & -10 \\
& K-factor (linear) & 1 & 0 & 0 \\
\hline SUI-5 & Delay $(\mu \mathrm{s})$ & 0 & 4 & 10 \\
$\left(\tau_{r m s}=2.842 \mu \mathrm{s}\right)$ & Power (dB) & 0 & -5 & -10 \\
& K-factor (linear) & 0 & 0 & 0 \\
\hline
\end{tabular}

Table III

Possible block interleaver sizes

\begin{tabular}{|cl||cc|}
\hline \multicolumn{2}{|c||}{ Sizes 1 } & \multicolumn{2}{c|}{ Sizes 2 } \\
\hline rows & columns & rows & columns \\
\hline 64 & 3 & 3 & 64 \\
32 & 6 & 6 & 32 \\
16 & 12 & 12 & 16 \\
8 & 24 & 24 & 8 \\
4 & 48 & 48 & 4 \\
2 & 96 & 96 & 2 \\
\hline
\end{tabular}

pattern (random permutation of integers 1 to 192) selected with a constraint on minimum allowed index spacing to be 6 sub-carriers is also studied.

\section{RESULTS}

In this section, we compare the performance of the spacefrequency coded systems with the selected interleavers against the performaces without interleaving. We also plot the results of two other configurations for comparative evaluation. One is by modelling the MIMO-OFDM system directly in the frequency domain with random fading along the sub-carriers (i.e. one where each sub-carrier undergoes fading independent from that of adjacent subcarriers). The other is a 3-tap channel with uniform delay spacing, equal power in each tap, and the maximum possible excess delay of $32 \mu \mathrm{s}$. This represents the highest frequency selective 3tap channel that can just be successfully compensated by the largest allowed guard time in the standard at the specified sampling rate.

The error rates are measured as Bit Error Rates (BER) and Packet Error Rates (PER), where each packet is defined as a codeword of the space-diversity code, and a packet error is counted when at least one bit error occurs in a packet (this PER is also known as Frame Error Rate - FER). Each codeword was constructed to span 192 QPSK symbols in each $T x$ antenna chain so that it could be transmitted by all the data sub-carriers of one OFDM symbol. This choice restricts only to frequency diversity, which is the main diversity resource in the channel. The temporal diversity, that can be extracted if codewords span multiple OFDM symbols, is negligible here because of the very low or no mobility assumption. The error rates are plotted against the effective 


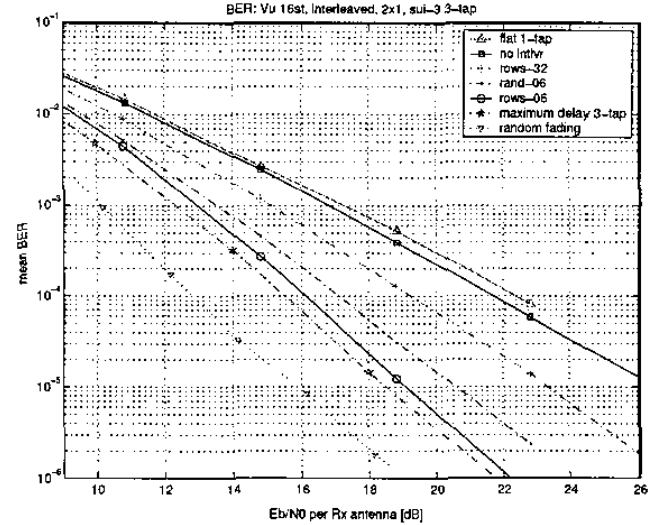

Fig. 3. SUI-3 channel, Vucetic 16-state code BER improvement

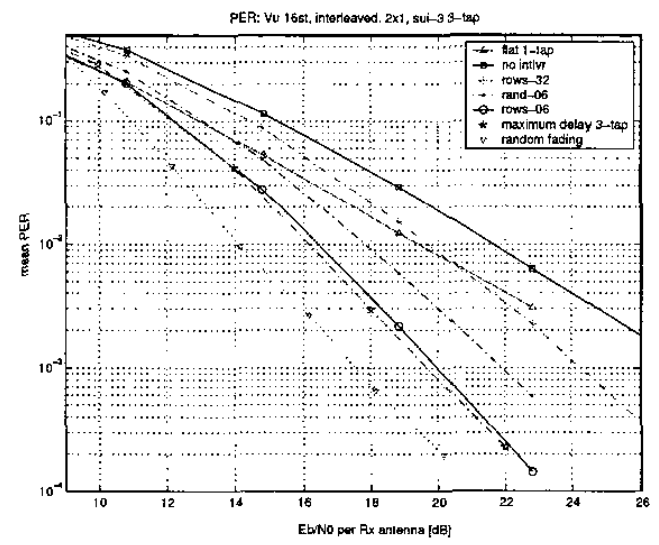

Fig. 4. SUl-3 channel, Vucetic 16-state code PER improvement

$\mathrm{Eb} / \mathrm{NO}$ (Bit energy to noise spectral density ratio) in each case.

The BER and PER of the Vucetic 16-state codes in SUl3 channel are in Figs. 3 and 4 respectively. It can be seen that the best performance is obtained with the $6 \times 32$ interleaver. When compared to the un-interleaved performance, it offers approximately $5 \mathrm{~dB}$ advantage in BER at $10^{-4}$ level and nearly $7 \mathrm{~dB}$ advantage in PER at $10^{-3}$ level. This performance is close to within $0.5 \mathrm{~dB}$ to that obtained by the fictitious maximally frequency selective channel. When compared to the purely independent fading across the frequencies, this performance is inferior by about $3 \mathrm{~dB}$ in BER and $2 \mathrm{~dB}$ in PER. However, it is an ideal case and this level of random fading is not possible in realistic channels where the number of taps are much less than the number of OFDM sub-carriers (or the size of the FFT operation). Encouragingly, the diversity slope is equalled in spite of the SNR shift.

Comparing the best interleaver with the random permutation

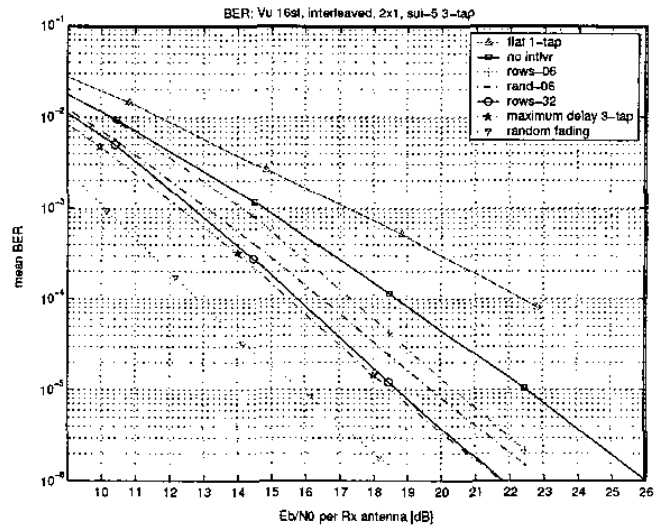

Fig. 5. SUI-5 channel, Vucetic 16-state code BER improvement

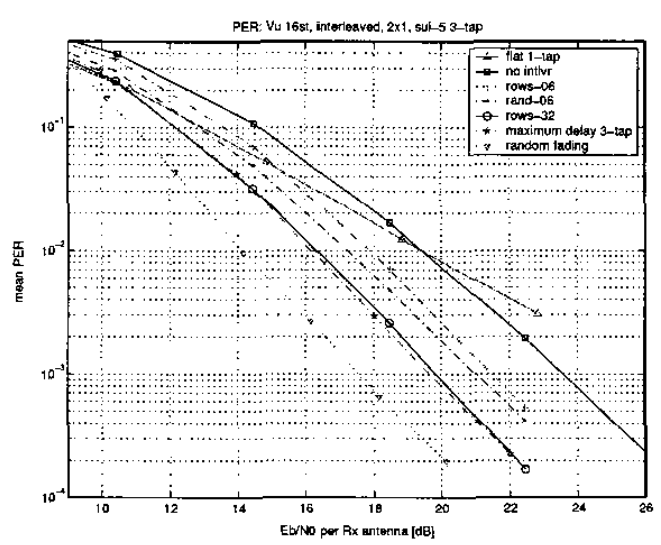

Fig. 6. SUl-5 channel, Vucetic 16-state code PER improvement

interleaver, we see a gain of $1 \mathrm{~dB}$ at BER of $10^{-4}$ and PER of $10^{-3}$. The interleaver of depth 32, which is the best for the SUI- 5 channel, is $3 \mathrm{~dB}$ and $4 \mathrm{~dB}$ worse off in BER of $10^{-4}$ and PER of $10^{-3}$ respectively. It is also worth noting that the worst interleaver $(64 \times 3$, which is not plotted here) of SUI-3 offered only upto $1 \mathrm{~dB}$ gain compared to no interleaving.

The performance of the same code as above (Vucetic 16state) in the SUI- 5 channel is shown in Figs. 5 and 6 as BER and PER. Similar observations are again made. However, the best interleaver in this case is the $32 \times 6$ interleaver, which is almost exactly matched also by the $12 \times 16$ interleaver (not shown in the plot to avoid clutter). The gain compared to uninterleaved case is estimated to be nearly $3 \mathrm{~dB}$ in BER at the $10^{-4}$ and $3.8 \mathrm{~dB}$ in PER at $10^{-3}$ levels. As in the SUI3 channel, here too the best performing interleaver is $3 \mathrm{~dB}$ away in BER and 2dB away in PER compared to the ideal independent fading of sub-carriers, but the diversity slope is completely matched. The random permutation interleaver is nearly $1 \mathrm{~dB}$ inferior to the best in both BER and PER. The 


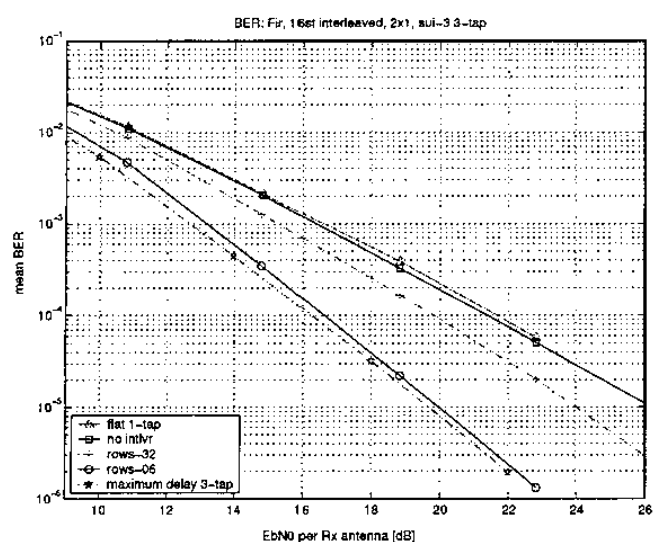

Fig. 7. SUI-3 channel, Firmanto 16-state code BER improvemen

best interleaver of SUI-3 channel is approximately $1.5 \mathrm{~dB}$ worse off here in SUI-5 channel.

Based on these observations, the fast-fading optimised Firmanto 16-state codes of [3] were studied with the important interleavers. Fig. 7 shows the BER in the SUI-3 channel. The un-interleaved performance is almost as bad as in the flat fading channel. Next, the $32 \times 6$ interleaver, which is the best for the SUI-5 channel, provides a $1.5 \mathrm{~dB}$ gain at $10^{-4}$. The best interleaver as seen before is the $6 \times 32$ which offers a $4.5 \mathrm{~dB}$ improvement at $10^{-4} \mathrm{BER}$, and a visibly increased diversity slope. It matches the performance of the maximally frequency selective 3-tap channel.

Fig. 8 illustrates the same Firmanto 16-state code's BER in the SUI-5 channel. Here the un-interleaved performance itself is $2.2 \mathrm{~dB}$ better than that of the flat fading case, with an improvement also in the diversity slope. The best interleaver of SUI- 3 offers nearly $2 \mathrm{~dB}$ gain, while the best of SUI5 offers a $3 \mathrm{~dB}$ gain, which equals the performance in the maximally frequency selective channel.

\section{CONCLUSION}

It is shown that appropriate interleaving can dramatically improve the error rate performance of space-frequency coding in frequency selective fading channels. This is important in a less frequency selective channel, where un-interleaved performance can be nearly as bad as in a frequency-flat channel which offers no frequency diversity. The results also highlight the most suitable interleaving depths for block interleavers to be used in HIPERMAN and 802.16a systems when operating under the two important channel types addressed. With these interleavers, the error performance in the SUI-3 and SUI-5 channels, which have highly contrasting degrees of frequency selectivity, are made to be almost equal. It is also found that good interleavers for one particular frequency selective channel can perform poorly in other channels. Based on the cases considered here, for

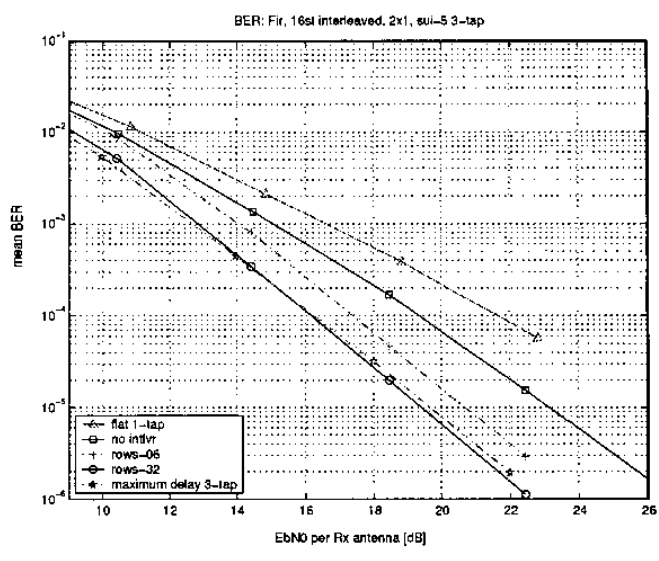

Fig. 8. SUI-5 channel, Firmanto 16-state code BER improvement

a highly frequency selective channel such as the SUI-5, good interleavers have relatively large index spacing (high interleaving depth) compared to the suitable ones for less frequency selective channel such as the SUI-3. Conversely, the degradation in using unsuitable interleaver is small in the highly frequency selective SUI-5 channel while it is considerably high in SUI-3. Thus, the choice of interleavers is critical in low delay-spread channels.

\section{ACKNOWLEDGEMENT}

The authors would like to thank the consortium members of the IST-STRIKE project, specially the members of WP4 (Thales Communications, CSEM, CEA LETI, CPK - University of Aalborg, and Nokia). R.R. would like to thank Dr. Peter Sweeney for useful discussions.

\section{REFERENCES}

[1] V. Tarokh, N. Seshadri, and A. R. Calderbank, "Space-time codes for high data rate wireless communications: performance criterion and code construction," IEEE Trans. Inf. Theory, vol. 44, no. 2, Mar. 1998, pp.744 765 .

[2] Zhuo Chen, Branka Vucetic, Jinhong Yuan, Ka Leong Lo, "Space-time trellis codes with two, three and four transmit antennas in quasi-static flat fading channels", ICC 2002 (IEEE International Conference on Communications, 2002), 28 April-2 May 2002, Vol. 3, pp. $1589-1595$.

[3] W. Firmanto, B.S. Vucetic, J. Yuan, "Space-time TCM with improved performance on fast fading channels," IEEE Communications Letters, Volume: 5 Issue: 4, Apr 2001, pp: $154-156$.

[4] Helmut Bolcskei, David Gesbert, Arogyaswami J. Paulraj, "Spacefrequency coded broadband OFDM systems," Proc. IEEE Wireless Communications \& Networking Conference (WCNC), Sept.2000.

[5] Roshano Roberts, Reza Hoshyar, M. Sadegh Fazel, and Rahim Tafazolli, "Performance of space-diversity trellis codes in HiperMAN and $802.16 \mathrm{a}$ broadband wireless access systems," In press, Proc. IEEE VTC-2004 spring, May 2004, Milano, Italy.

[6] Е. Mino Diaz et al., "4G scenarios and system requirements", Deliv erable D2.1, IST-2001-38354 STRIKE project, April 2003.

[7] ETSI TS $102.177 \mathrm{v}<1.1 .1>(2003-11)$, "Broadband Radio Access Networks (BRAN); HIPERMAN; Physical (PHY) Layer".

[8] IEEE 802.16 Broadband Wireless Access Working Group <http://ieee802.org/16>, "Channel Models for Fixed Wireless Applications", IEEE 802.16.3c-01/29r2, 2001-07-05. 